\title{
Co-expression of autophagic markers following photodynamic therapy in SW620 human colon adenocarcinoma cells
}

\author{
BARBARA ZIÓłKOWSKA, MARTA WOŹNIAK and PIOTR ZIÓŁKOWSKI \\ Department of Pathology, Wrocław Medical University, 50-368 Wrocław, Poland
}

Received January 31, 2016; Accepted May 31, 2016

DOI: $10.3892 / \mathrm{mmr} .2016 .5541$

\begin{abstract}
Photodynamic therapy (PDT) is a minimally invasive cancer treatment. It involves the combination of a photosensitizer and light of a specific wavelength to generate singlet oxygen and other reactive oxygen species that lead to tumor cell death. Autophagy is one of the pathways that tumor cells undergo during photodamage and it is common in photodynamic therapy. The aim of this study was to examine the effect of in vitro PDT on the expression of autophagy-related proteins, autophagy related 7 (Atg7), light chain 3 (LC3) and Beclin-1. Human SW620 colon carcinoma cells were treated with 5-aminolevulinic acid (ALA)-based PDT at a dose of $3 \mathrm{mM}$. The irradiation was performed using $4.5 \mathrm{~J} / \mathrm{cm}^{2}$ total light and a fluence rate of $60 \mathrm{~mW} / \mathrm{cm}^{2}$. Autophagy was evaluated by immunocytochemistry using specific antibodies to Atg7, Beclin-1 and LC3. The evaluation was repeated at several time points $(0,4,8$ and $24 \mathrm{~h})$ following irradiation. The induction of autophagy was observed directly following the 5-ALA-mediated PDT procedure with the strongest expression of autophagy-related proteins at 4 and $8 \mathrm{~h}$ after irradiation as demonstrated using immunocytochemistry. It was characterized by significantly increased expression of Beclin-1, Atg7 and LC3. To the best of our knowledge this is the first study to analyze Beclin-1, Atg7 and LC3 expression in a PDT-related experiment. This study enhances the understanding of the role of autophagy in PDT, which may contribute to better and more effective tumor responses to this therapy.
\end{abstract}

\section{Introduction}

Photodynamic therapy (PDT) is a method of treatment that involves selective photosensitization of malignant cells, usually by means of porphyrins, porphyrin analogs or other agents with suitable photophysical qualities. The use of

Correspondence to: Professor Piotr Ziółkowski, Department of Pathology, Wrocław Medical University, ul. Marcinkowskiego 1, 50-368 Wrocław, Poland

E-mail: ziolkows@interia.pl

Key words: photodynamic therapy, autophagy, cell death, cancer, colon cancer cells photodynamic therapy results in direct cell damage or induction of cell death (1). Certain photosensitizers accumulate in the endothelial cells of vascular tissue. This leads to the initiation of thrombogenic sites within the vessel and results in a cascade of responses, including platelet aggregation, leukocyte adhesion, the release of vasoactive molecules, increases in vascular permeability and vessel constriction. The first step in the photodynamic process is associated with the localization of the photosensitizing agent in subcellular loci. These can be highly specific or quite broad, and have been reported to include the endoplasmic reticulum, mitochondria, Golgi, lysosomes and plasma membranes (2). The majority of photosensitizers are hydrophobic and are thus attracted to membranes.

Following incubation with a photosensitizing agent, cells are exposed to light at a wavelength corresponding to an absorbance band (most commonly at a longer wavelength of red visible light 620-690 nm). As a result of a photophysical reaction, numerous reactive oxygen species (ROS) are released. The singlet molecular oxygen is the most unstable of all ROS and can translocate no further than microns from the site of their formation. Therefore, photodamage can be highly precise. Other ROS formed downstream from singlet oxygen are able to migrate a further distance from the primary site $(3,4)$. As they are highly reactive with cellular components, such as proteins, lipids and DNA, ROS are a source of cytotoxicity. If the ROS are not neutralized by cellular detoxifying and antioxidant enzymes, they may lead to the destruction of the cellular machinery as a result of oxidative stress (3). The role of autophagy and apoptosis appears to be crucial at this stage.

Autophagy is a complex 'self-eating' system involving degradation of dispensable or impaired cellular constituents by the actions of lysosomes. It primarily functions as a cell survival, adaptive mechanism in conditions of nutrient starvation, infections or protein aggregate-induced stress. In mammalian cells, autophagy is one of the main clearance systems for ROS-damaged organelles or irreparable oxidized cytosolic proteins. Data show that ROS can activate autophagy; the consequences vary from protection to the promotion of autophagic cell death (5).

Three different types of autophagy are identified: Macroautophagy (MA), microautophagy and chaperone-mediated autophagy (CMA). Initially, the formation of an isolation membrane (phagophore) curves around a part of the cytoplasm 
and forms a closed double membrane vesicle (autophagosome) (6). During its maturation, microtubule-associated protein light-chain 3 (LC3-I) is detached and then linked with phosphatidylethanolamine to form LC3-II, which is recruited into an autophagosomal membrane. This action is mediated by the autophagy-related (Atg) proteins Atg7 and Atg3 (7). Autophagosomes fuse with lysosomes to form autolysosomes. During the degradation of the intra-autophagosomal components the lipidated LC3-II is also destroyed. Thus, LC3-II is a hallmark of autophagy (8-10).

In the settings of cancer, autophagy has a double role depending on the stages of cancer. It can support tumor suppression (at the early stage of tumorigenesis) or promote tumor progression (at the advanced stages of tumor) (11). The tumor suppressor mechanism is probably connected with the upregulation of the class I phosphatidylininositol 3-kinase (PI3K) pathway in cancer cells and may correspond with Beclin-1 function, deletion of which is relatively common in breast, ovarian and prostate cancer (12). Autophagy upregulation upon cellular detachment from the extracellular matrix also sustains cell viability in metastasizing cells (12). Knockdown of essential autophagy genes in tumor cells may potentiate the induction of cell death.

\section{Materials and methods}

Photosensitisation. In all experiments, $3 \mathrm{mM}$ precursor-5-aminolevulinic acid (5-ALA; Sigma-Aldrich, Munich, Germany) was used for PDT, the dose was established in our previous studies (Ziółkowski P et al, unpublished data).

The light source at a wavelength of $630+/-20 \mathrm{~nm}$ (obtained with a bandpass filter), was achieved with the Penta lamps Teclas (Teclas, Lugano, Switzerland). The total light dose was $4.5 \mathrm{~J} / \mathrm{cm}^{2}$ and the fluence rate was $60 \mathrm{~mW} / \mathrm{cm}^{2}$, which was applied $4 \mathrm{~h}$ after administration of 5-ALA.

Cell cultures. SW620 colon adenocarcinoma cells obtained from the Institute of Immunology and Experimental Therapy (Wrocław, Poland) were maintained in DMEM/F12 (Gibco, Thermo Fisher Scientific, Inc., Waltham, MA, USA) supplemented with $10 \%$ fetal bovine serum (FBS) and $1 \%$ glutamine (GlutaMax, Thermo Fisher Scientific, Inc.) in $5 \% \mathrm{CO}_{2}$ at $37^{\circ} \mathrm{C}$ and a $95 \%$ humidified atmosphere. Cells were counted in suspensions using a Countess Automated Cell Counter (Invitrogen, Thermo Fisher Scientific, Inc.) and then seeded at a density of $5 \times 10^{4}$ and $4 \times 10^{5}$ cells per well in 96- and 12 -well culture plates (TPP, Trasadingen, Switzerland), respectively, and cultured for another $48 \mathrm{~h}$. Subsequently medium was replaced by DMEM/F12 with 5-ALA (3 mM) for $4 \mathrm{~h}$. After incubation, the medium was replaced with FBS-free DMEM/F12 and irradiation was performed with red light. After PDT, medium was changed again to DMEM/F12.

Each experiment was conducted in triplicate and cells were divided into 4 groups: Cells treated with PDT, cells treated with precursors only, cells treated with light only or untreated control cells.

Cell viability assays. Cell proliferation and PDT cytotoxicity were determined prior to immunocytochemistry and western blot analysis by a colorimetric assay with 3-(4,5-dimethylthiazol-2-yl)-2,5-diphenyltetrazolium bromide (MTT). It is based on the reduction of a yellow soluble tetrazole to an insoluble purple formazan in respiring cells. Cells were plated at a density of $5 \times 10^{4}$ cells per well in a 96 -well plate and cultured. Then, control cells and cells incubated with the precursor photosensitizer were irradiated with light. Following irradiation $(24 \mathrm{~h})$, cells were extensively washed with phosphate-buffered saline (PBS) and incubated at $37^{\circ} \mathrm{C}$ for $4 \mathrm{~h}$ with MMT/phenol-red free Dulbecco's modified Eagle's medium (DMEM)/F12 (v/v=1:9) (Gibco, Thermo Fisher Scientific, Inc.). The medium was removed and $100 \mu \mathrm{l}$ dimethyl sulfoxide (DMSO, Sigma-Aldrich) was added to each well for $5 \mathrm{~min}$. The optical absorbance (A) was estimated at $490 \mathrm{~nm}$ using a BioTek ELX800 multi-well reader (BioTek, Winooski, VT, USA). The absorbance in the control group was counted as $100 \%$ cell viability. The percentage of viable cells (VC) was evaluated according to: VC $(\%)=(\mathrm{A}$ of experimental group / A of control group) x 100. Cell viability was estimated to be $95 \%$.

Cell cultures and photosensitization conditions for immunocytochemistry. Cells were plated at a density of $5 \times 10^{4}$ cells per well in 3-well (each well of $14 \mathrm{~mm}$ in diameter), epoxy-coated, diagnostics glass slides (Menzel-Glaser; Thermo Fisher Scientific Inc.) and placed into humidified Petri dishes. The growth and treatment conditions in the following section.

Cell cultures and photosensitization conditions for western blot analysis. Cells were seeded in plastic 12-well plates $\left(4 \times 10^{5}\right.$ cells per well) and incubated with 5 -ALA for $4 \mathrm{~h}$ in the medium with $5 \%$ FBS without phenol red. Thereafter the medium was replaced with the medium without serum and phenol red and cells were exposed to the light source. After irradiation, the medium was replaced with DMEM/F12. Following the 24-h incubation the medium was removed by double rinsing with phosphate-buffered saline (PBS) and the cells were lysed. Following the 24-h incubation western blot analysis was performed.

Western blotting. Cells were rinsed twice with pre-cooled PBS and treated with $200 \mu \mathrm{l} /$ well lysis buffer [4\% sodium dodecyl sulfate (SDS), 0.1 M DTT in 0.1 M Tris/HCl buffer, pH 7.6] containing protease and phosphatase inhibitors (1\% cocktails, Sigma-Aldrich). Samples were centrifuged at 15,000 x g for $30 \mathrm{~min}$. The supernatant was collected and the protein concentration was determined by a spectrophotometer at $280 \mathrm{~nm}$ (PicoDrop 2000; Thermo Fisher Scientific, Inc.). The protein extracts $(25 \mu \mathrm{g})$ were separated by $4-12 \%$ SDS-polyacrylamide gel electrophoresis (Invitrogen, Thermo Fisher Scientific Inc.) and transferred to nitrocellulose membranes (Amersham Hybond; GE Healthcare Life Sciences, Little Chalfont, USA). The membrane was blocked with PBS with $0.1 \%$ Tween-20 (pH 7.6 with 10\% goat serum; Sigma-Aldrich) for $1 \mathrm{~h}$ at room temperature. Subsequently, the membranes were incubated with the primary antibodies: Rabbit polyclonal anti-LC3 diluted 1:350 (cat. no. HPA052484; Sigma-Aldrich); rabbit polyclonal anti-Atg7 diluted 1:500 (cat. no. sc-33211; Santa Cruz Biotechnology, Inc., Santa Cruz, CA, USA) and rabbit polyclonal anti-Beclin-1 diluted 1:250 (cat. no. HPA028949; Sigma-Aldrich), overnight at $4^{\circ} \mathrm{C}$. The membranes were then 
Table I. Immunoreactivity score calculation method.

\begin{tabular}{|c|c|c|c|c|c|}
\hline \multicolumn{2}{|c|}{ Intensity of staining } & \multicolumn{2}{|c|}{ Stained cells (\%) } & \multicolumn{2}{|c|}{ IRS } \\
\hline Score & Staining & Score & $\%$ stained & Score & Reaction \\
\hline 0 & Negative & 0 & $<5$ & 0 & Negative \\
\hline 1 & Weak & 1 & $5-25$ & $1-2$ & Weak \\
\hline 2 & Moderate & 2 & $25-75$ & $3-4$ & Moderate \\
\hline 3 & Strong & 3 & $>75$ & $6-9$ & Strong \\
\hline
\end{tabular}

IRS was calculated by multiplying the staining intensity value by the percentage of stained cells. IRS, immunoreactivity score.

washed three times with PBS and incubated with horseradish peroxidase-labeled secondary goat anti-rabbit antibody (cat. no. sc-2030; Santa Cruz Biotechnology, Inc.) for $1 \mathrm{~h}$ at room temperature and thereafter washed again three times with PBS. The final color reaction was detected using enhanced colorimetric western blotting visualization reagents and the DAB Enhanced Liquid Substrate System for Immunochemistry (Sigma-Aldrich). The image of the specific protein bands was documented by Bio-Rad equipment (MolecularImager Gel Doc TMXR+) and relevant software Image Lab Software v4.1 (Bio-Rad Laboratories, Hercules, CA, USA).

To normalize the loading differences a monoclonal $\beta$-actin antibody against the housekeeping control $\beta$-actin was used.

Immunocytochemistry. Immunocytochemistry was performed using the LSAB+ method (LSAB+ System-HRP; DAKO, Glostrup, Denmark). At indicated times (0, 4, 8 and $20 \mathrm{~h}$ ) following light exposure, cells were fixed in $4 \%$ paraformaldehyde at $4^{\circ} \mathrm{C}$ for $10 \mathrm{~min}$ and subsequently washed with PBS (0.1 M phosphate buffer, pH 7.4; and $0.15 \mathrm{M} \mathrm{NaCl}$ ). Glass slides containing cells were incubated with an endogenous peroxidase blocking buffer and then with a protein blocking buffer. Next, primary antibodies (anti-Beclin-1, dilution 1:100; anti-LC3, dilution 1:250; Sigma Aldrich and anti-Atg7, dilution 1:100; from Santa Cruz Biotechnology, Inc.) were added for overnight incubation at $4^{\circ} \mathrm{C}$. On the following day, the slides were incubated for $15 \mathrm{~min}$ with biotinylated antibody and streptavidin-HRP, respectively. Then they were twice rinsed with PBS and stained using 3,3'-diaminobenzidine in chromogen solution. Sections were counterstained with Mayer's hematoxylin and then dehydrated in graded alcohol, cleared in xylene and mounted with xylene based mounting medium. For the negative staining control, the primary antibody was omitted. Images were acquired using a light microscope fitted with digital camera (Nicon Eclipse 80i with camera DS-Fil-U2, Amsterdam, Netherlands) at magnifications of x100 and x200.

The protein expression of Beclin-1, LC3 and Atg7-positive cells in the glass slides was defined using the immunoreactivity score (IRS) as shown in Table I.

Statistical analysis. Statistical analysis was performed using multiple comparisons analysis of variance followed by Tukey's test. The software used for the analysis was STATISTICA v.10
(StatSoft, Inc., Tulsa, OK, USA). $\mathrm{P}<0.05$ was considered to indicate a statistically significant difference.

\section{Results}

Cell viability. A relatively poor cytotoxicity of SW620 cell lines was observed following PDT. The cell viability estimated by the MTT test after PDT was $95 \%$.

Immunocytochemistry. The key finding was that following PDT, three markers of autophagy were strongly expressed in treated cells, and this was shown to be greater than that in the control cells. Prior to application of 5-ALA and irradiation with the light source Beclin-1 was not identified (IRS=0). Fig. 1A shows cancer cells from the control group (no 5-ALA, no light), where no staining against Beclin-1 was observed. Fig. 1B shows the effect of 5-ALA-PDT at $0 \mathrm{~h}$, directly following treatment when the expression of Beclin-1 was found to be weak (IRS=1), while after $4 \mathrm{~h}$ it was not identified to change significantly (IRS=3; P<0.001), (Fig. 1C). Fig. 1D shows that the PDT effects on Beclin-1 expression were strongest, with diffuse cytoplasmic staining $8 \mathrm{~h}$ after treatment $(\mathrm{IRS}=6 ; \mathrm{P}<0.001)$.

PDT also resulted in an increase in the expression of the LC3 protein, as confirmed by immunocytochemical staining at different time points. Fig. 1E shows an effect of PDT on SW620 cells in the control group (no 5-ALA, no light) as the cancer cells present a weak diffuse cytoplasmic staining (IRS=2). At the first time point directly after the PDT $(0 \mathrm{~h})$ the intensity of staining and number of stained cells remained the same $($ IRS $=2)$ (Fig. 1F). At the next time points, 4 and $8 \mathrm{~h}$ following PDT, the intensity of staining and number of stained cells significantly increased. Fig. $1 \mathrm{G}$ and $\mathrm{H}$ show the effects of PDT at 4 (IRS=9) and $8 \mathrm{~h}$ (also IRS=9), respectively (both $\mathrm{P}<0.001$ ).

The protein expression of Atg7 was also increased following PDT. Fig. 2A shows the results of immunocytochemical staining of SW620 cells in the control group (no 5-ALA, no light). Contrary to Beclin-1 and LC3, strong expression was observed (IRS=6). Directly after irradiation at time point $0 \mathrm{~h}$ the expression of Atg7 was strong (Fig. 2B), (IRS=9; $\mathrm{P}<0.01$ ), and remained strong at $4 \mathrm{~h}$ following PDT (IRS=9), (Fig. 2C). Later at $8 \mathrm{~h}$ this expression slightly decreased but it was still strong (IRS=6), (Fig. 2D).

After PDT (24 h) strong expression of Beclin-1 protein (IRS=6; $\mathrm{P}<0.001$; Fig. 2E) and LC3 protein (IRS=6; $\mathrm{P}<0.001$ ); Fig. 2F) was observed although, the expression of LC3 showed 

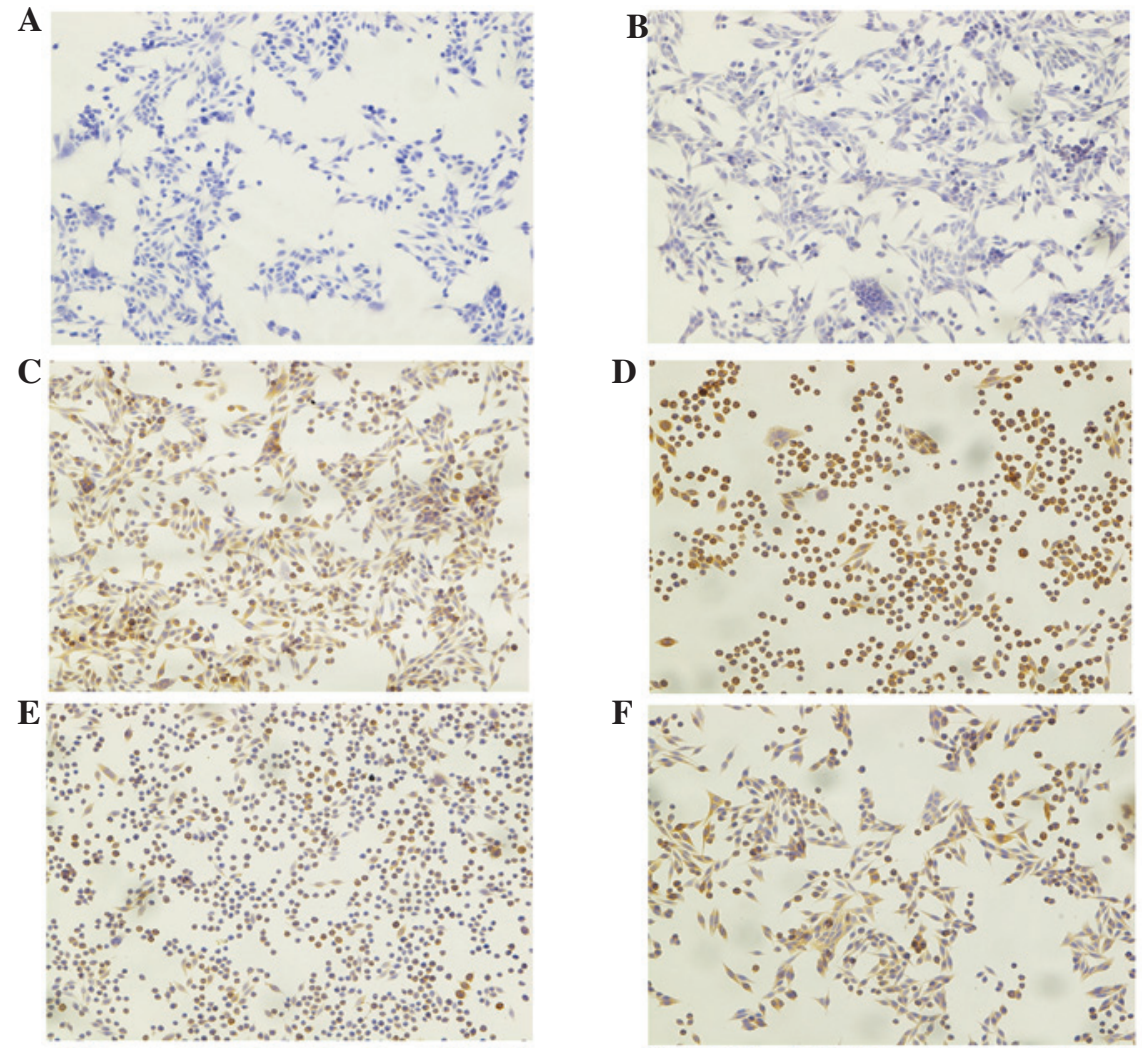

F
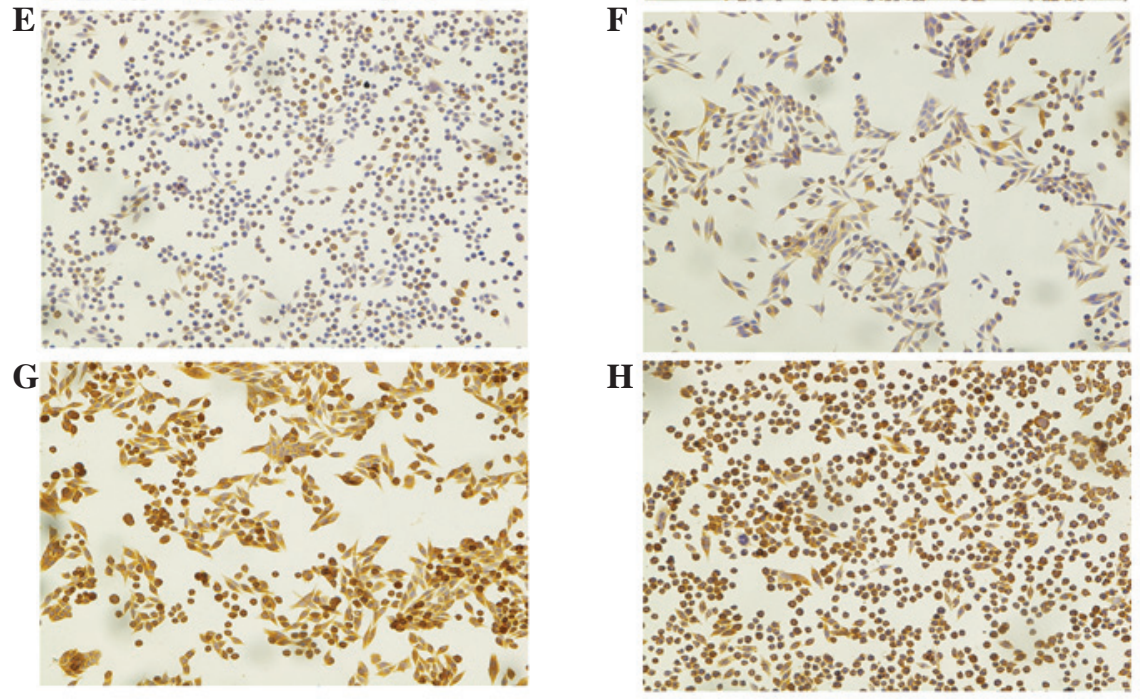

Figure 1. Immunocytochemistry of Beclin-1 and LC3 in SW620 human colon adenocarcinoma cells. (A-D) Beclin-1 expression. (A) Cells from the control group, neither 5-ALA nor light was applied. No staining was observed (IRS=0). (B) Cells from group treated with $3 \mathrm{mM} 5$-ALA and $4.5 \mathrm{~J} / \mathrm{cm}^{2}$ light. Very weak cytoplasmic staining was found at $0 \mathrm{~h}$ directly after PDT (IRS=1). (C) Cells from the PDT treated group at $4 \mathrm{~h}$ show marginal increase of staining (IRS=3). (D) Cells from the PDT-treated group at $8 \mathrm{~h}$ showed the most intense and diffuse staining (IRS=6). (E-H) LC3 expression. (E) Cells from the control group, neither 5-ALA nor light was applied. Weak cytoplasmic staining was observed in cancer cells (IRS=2). (F) Cells from the group treated with 3 mM 5-ALA and $4.5 \mathrm{~J} / \mathrm{cm}^{2}$ light. A weak cytoplasmic staining was found at $0 \mathrm{~h}$ after PDT (IRS=2). (G) Cells treated with PDT (time point $4 \mathrm{~h}$ ). Strong, diffuse cytoplasmic staining is observed in the majority of cells (IRS=9). (H) Cells treated with PDT (time point $8 \mathrm{~h}$ ). Strong diffuse staining in cell cytoplasm was found in a large number of cells (IRS=9). Hematoxylin counterstained. Magnification, x200. LC3, light chain 3; 5-ALA, 5-aminolevulinic acid; PDT, photodynamic therapy; IRS, immunoreactivity score.

the tendency to decrease. The expression of Atg7 protein decreased to moderate (Fig. 2G). In addition, the cell population was markedly decreased following $24 \mathrm{~h}$.

All microscopic sections were additionally evaluated by two pathologists. The results presented on the bar chart show the arithmetic mean of the immunoreactivity scores for each protein at the indicated time point. It was demonstrated that the highest expression of all autophagy-related proteins was observed from 4 to $8 \mathrm{~h}$ and then decreased at $24 \mathrm{~h}$ (Fig. 3). The bar chart shows that there was a significant increase of Beclin-1 expression from 0 to $8 \mathrm{~h}$, which remained stable until $24 \mathrm{~h}$ following irradiation $(\mathrm{P}<0.001)$. Conversely, Atg7 expression (IRS=9) was high immediately post 5-ALA-PDT and decreased from $8 \mathrm{~h}(\mathrm{P}<0,001)$. The LC3 expression was visible at $0 \mathrm{~h}(\mathrm{IRS}=2)$ and significantly increased at 4 and $8 \mathrm{~h}$ $($ IRS=9) $(\mathrm{P}<0.001)$ (Fig. 3).
Western blot analysis. Western blot analysis was performed directly following light exposure $(0 \mathrm{~h})$, in order to detect the expression of Beclin-1 and Atg7, and the conversion of LC3 I to LC3 II, respectively. $\beta$-actin served as an internal control. A marked difference between samples incubated with 5-ALA only (lane 1), cells irradiated only (lane 2) and cells treated with 5-ALA-PDT (lane 3) compared with the control cells, respectively. The activation of autophagic cell death was strongest in the 5-ALA-PDT-treated SW630 cells (lane 3) (Fig. 4):

\section{Discussion}

Although autophagy has been extensively investigated, little was known regarding its molecular mechanism until the identification of autophagy-related genes in yeast (13). 

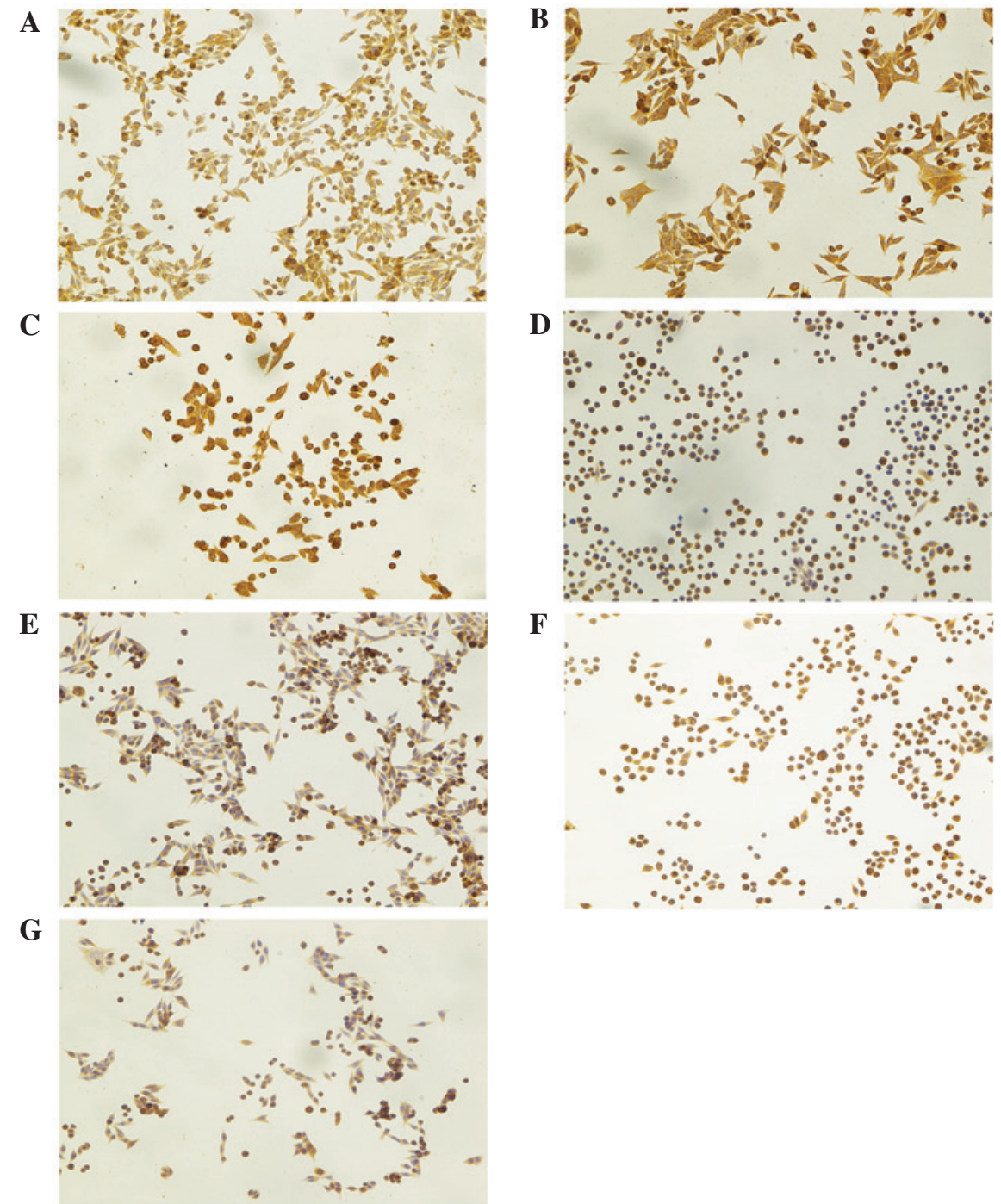

Figure 2. Immunocytochemistry of Atg7, Beclin-1 and LC-3 in SW620 human colon adenocarcinoma cells. (A-D) Atg7 expression. (A) Cells from the control group, neither 5-ALA nor light was applied. Strong staining was found in cancer cells (IRS=6). (B) Cells from group treated with $3 \mathrm{mM} \mathrm{5-ALA} \mathrm{and} \mathrm{4.5} \mathrm{J/cm²}$ light. Very strong cytoplasmic staining was found at $0 \mathrm{~h}$ after PDT (IRS=9). (C) Cells treated with PDT (time point $4 \mathrm{~h}$ ). Very strong, diffuse cytoplasmic staining was found in cancer cells (IRS=9). (D) Cells treated with PDT (time point $8 \mathrm{~h}$ ). Strong diffuse staining in cell cytoplasm was observed in a large number of cells $(I R S=6)$. (E-G) The results of immunocytochemical staining in SW620 human colon adenocarcinoma cells against three studied proteins at $24 \mathrm{~h}$ from PDT. (E) Beclin-1 expression. Strong staining was found in cancer cells (IRS=6), (F) LC-3 expression. Cells show an intense diffuse staining (IRS=6), (G) Atg7. Moderate cytoplasmic staining was observed (IRS=4). Hematoxylin counterstained. Magnification, x200. Atg7, autophagy-related gene 7; LC3, light chain 3; 5-ALA, 5-aminolevulinic acid; PDT, photodynamic therapy; IRS, immunoreactivity score.

Autophagy is a cardinal cellular mechanism that involves the degradation and digestion of intracellular constituents by lysosomes. It is also involved in controlling inflammation (1-3). Autophagosome formation is controlled by protein complexes including the coiled-coil myosin-like BCL2-interacting protein 1 (Beclin-1) complex. The autophagy process is initiated by the regulation of protein complexes composed of $>30$ Atg proteins and autophagic adaptor LC3 protein (14-20). In the present study the expression of all the above proteins were investigated in terms of photodynamic therapy. To the best of our knowledge, this is the first study to evaluate Beclin-1, Atg7 and LC3 in one PDT-based experiment at several time points within $24 \mathrm{~h}$ of observation.

To date, 30 Atg genes have been identified. The corresponding gene products comprise the 'core' machinery (21) that coordinates the specific steps in the autophagic pathway, including two ubiquitin-like conjugation systems, Atg12 and Atg8 (22-24). Atg12 is activated by an E1-like enzyme, Atg7 (25), which was one of our main targets investigated, and finally conjugated to Atg5 in a reaction similar to ubiquitination. Atg7 can also activate Atg8 thereby participating in the Atg8 conjugation system. In mammalian cells, Atg7 is essential for the autophagy conjugation system, formation of autophagosomes, and starvation-induced degradation of proteins and organelles (26).

Protection against cell death and sensitization to other (non-PDT) stressors have been observed when Atg7 was knocked down. Kessel and Reiners (5) and Kessel and Arroyo (27) depleted Atg7 in L1210 murine leukemia cells by short hairpin RNA knockdown, and noted that the deficient cells were more sensitive than Atg7-replete cells to the lethal effects of a low PDT dose. This suggested that autophagy served a survival function in leukemia L1210 cells. By contrast, other laboratories have reported that Atg7 knockdown protected 


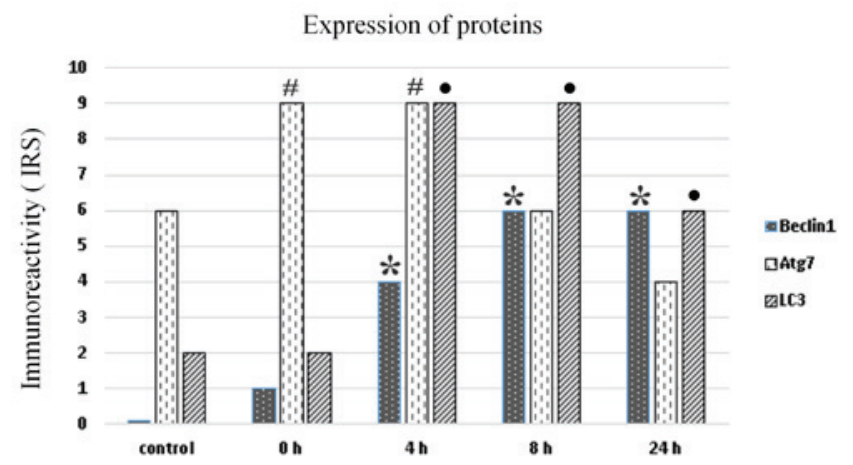

Figure 3. Bar chart showing the IRS of Beclin-1, Atg7 and LC3 in SW620 human colon adenocarcinoma cells treated with 5-ALA-PDT and stained at the indicated time points following irradiation. Beclin-1 expression was slowly but significantly increase from 0 to $8 \mathrm{~h}$ and remained stable $24 \mathrm{~h}$ after irradiation. Conversely, Atg7 was highly expressed (IRS=9) immediately post 5-ALA-PDT and decreased at $8 \mathrm{~h}$. The LC3 expression was visible at $0 \mathrm{~h}($ IRS=2) and increased at 4 and $8 \mathrm{~h}$ (IRS=9). IRS, immunoreactivity score; Atg7, autophagy-related gene 7; LC3, LC3, light chain 3; 5-ALA-PDT, 5 -aminolevulinic acid photodynamic therapy. "P<0.001 compared with the LC3 control. " $\mathrm{P}<0.001$ compared with the Atg7 control. ${ }^{\bullet} \mathrm{P}<0.001$ compared with the Beclin-1 control.
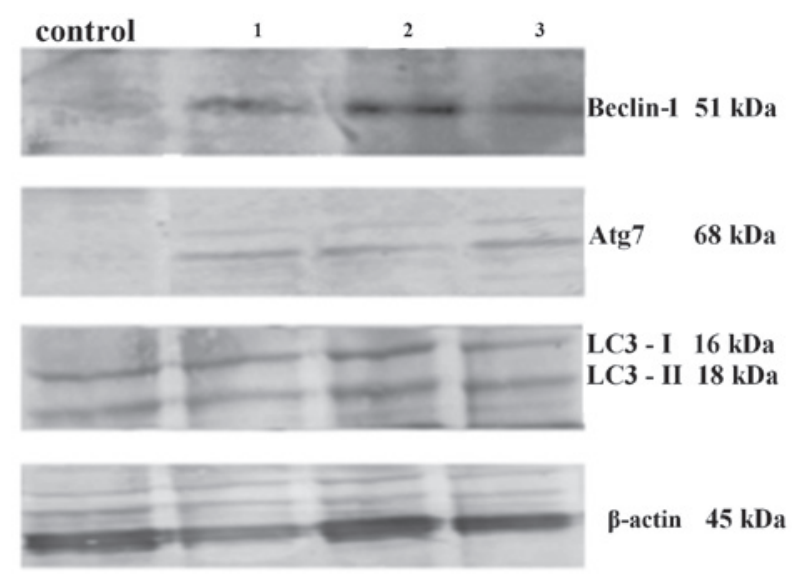

Figure 4. Western blot analysis. SW620 human colon adenocarcinoma cells were treated with $3 \mathrm{mM} \mathrm{5-ALA}$ for $4 \mathrm{~h}$, and then irradiated with a light dose of $4.5 \mathrm{~J} / \mathrm{cm}^{2}$. The cell lysates were collected at $0 \mathrm{~h}$ after 5-ALA-PDT. The control lane is cells without treatment. Lane 1, SW620 cells were subjected to 5-ALA only; lane 2, SW620 cells were irradiated only; and lane 3, SW620 cells treated with 5-ALA-PDT. Atg7, autophagy-related gene 7; LC3, LC3, light chain 3; 5-ALA-PDT, 5-aminolevulinic acid photodynamic therapy.

against cell death and similarly, it has been shown that chemical inhibitors of autophagy, 3-methyladenine (3-MA) and wortmannin, provided greater protection against loss of viability to apoptosis-deficient than to apoptosis-competent MCF-7 cells.

Another noteworthy difference between observations made by Xue et al (28) and those of Kessel and Reiners (5), is associated with the ability of the cells to generate LC3-II in response to PDT when expression of Atg7 is deficient. In the present study, strong immunocytochemical expression of Atg7 was observed following PDT, which increased to IRS=9 directly after treatment, and remained stable with a marginal decrease at $24 \mathrm{~h}$.

Beclin-1, the mammalian homolog of Atg6 in yeast, is a key member of the PI3K initiation complex responsible for initiating formation of the phagophore and was shown to be induced by PDT as determined by immunocytochemistry. In the present study a strong expression of Beclin-1 was observed, which was correlated with the initiation of autophagy. Beclin-1 binds Vps34 and Vps15 to form the core of the initiation complex (29), which can interact with other positive regulators of autophagy, such as Atg14 or can be disrupted by interaction with Bcl-2 to inhibit autophagy (30). The initiation complex also interacts with LC3 to increase the autophagic flux and early autophagosome formation (31). In the present study, no significant increase in LC3 expression was identified directly following PDT by means of immunocytochemistry. However, LC3 expression rapidly increased at $4 \mathrm{~h}$ and then remained high at 8 and $24 \mathrm{~h}$.

ROS are common by-products of the cellular metabolism and serve as essential signaling mediators in a variety of processes, including proliferation, senescence, ageing and carcinogenesis (1). When overproduced, ROS can directly affect cellular functions by oxidizing vital molecules that are crucial for cellular integrity, thereby causing cell death.

Recently, ROS have also emerged as signaling mediators in MA (32-34), a major lysosomal pathway for 'in bulk' removal of entire portions of the cytoplasm, including organelles (5). Although MA can selectively remove damaged or unnecessary organelles (e.g. mitochondria, peroxisomes and endoplasmic reticulum), it is considered an unselective degradation process of soluble cytosolic proteins or aggregates. By contrast, CMA is a selective pathway for protein-by-protein removal, based on the recognition of proteins exposing a KFERQ-related targeting sequence. Furthermore, it has been shown that CMA is the dominant cytoprotective pathway in PDT, whereas it is dispensable for ER stress-induced cell death (35).

In a study by Dewaele et al (32) findings indicated that along with increased apoptosis, attenuation of MA by 3-MA or Atg 5 knockdown enhanced the accumulation of ROS-damaged proteins in the photosensitized cells. This strongly suggests that MA participates in the removal of ROS-damaged cytoplasmic components and by doing so limits PDT-mediated injury (35). This mechanism could explain numerous failures of PDT and poor cytotoxicity of SW620 cells observed in our study.

The immunohistochemical analysis in our study showed that the level of Beclin-1 gradually increased whereas it remained unaltered during the time course of other experiments (3). In concordance with the study by Reiners et al (3) LC3 expression was increased with peak expression at later time points; $24 \mathrm{~h}$ (3) or $8 \mathrm{~h}$ in the present study. Notably, not all autophagy-related proteins undergo photodamage in PDT protocols. An examination of the effects of an LD90 PDT dose on Beclin-1, Atg5 and Atg7 revealed no significant photodamage (3). These results were obtained with L1210 cells and the photosensitizer benzoporphyrin derivative monoacid ring $\mathrm{A}$, which localizes preferentially to the mitochondria and does not accumulate in lysosomes. Likewise, neither Beclin-1 nor Atg5 were photodamaged in PDT protocols utilizing hypericin-photosensitized HeLa cells and mouse embryo fibroblasts (3).

After $24 \mathrm{~h}$, in cultures treated with PDT alone, the apoptotic cells were no longer observed and surviving cells were swollen and filled with vesicles (36). A similar effect was observed in the present study, however a part of the cell population 
visibly shrunk after $24 \mathrm{~h}$. As the induction of autophagy is a common response in PDT protocols, it appears unlikely that proteins responsible for the assembly of autophagosomes are PDT targets. Indeed, as reported in a number of studies, key autophagic proteins, such as Beclin-1, Atg5 and Atg7 appear to be unaffected in PDT protocols employing endoplasmic reticulum and mitochondrial sensitizers (3).

In conclusion, in the present study it was demonstrated that autophagy is involved in cell death induced by 5-ALA-PDT. The results showed expression of autophagy-related proteins shortly following PDT and a significant increase in the levels of these proteins in the following hours. The role of autophagy, particularly in PDT remains unclear and further investigation is required to determine the most effective parameters of PDT leading to the highest cytotoxic effects on cancer cells.

\section{References}

1. Dougherty TJ, Gomer CJ, Henderson BW, Jori G, Kesel D, Korbelik M, Moan J and Peng Q: Photodynamic therapy. J Natl Cancer Inst 90: 889-905, 1998 .

2. Kessel D: Correlation between subcellular localization and photodynamic efficacy. J Porph Phtalo 8: 1009-1014, 2004.

3. Reiners Jr JJ, Agostinis P, Berg K, Oleinick NL and Kessel D: Assesing autophagy in the context of photodynamic therapy. Autophagy 6: 7-18, 2010.

4. Castano AP, Demidiva TN and Hamblin MR: Mechanisms in photodynamic therapy: Part two-cellular signalling, cell metabolism and modes of cell death. Photodiagnosis Photodyn Ther 2: 1-23, 2005.

5. Kessel D and Reiners JJ Jr: Apoptosis and autophagy after mitochondrial and endoplasmic reticulum photodamage. Photochem Photobiol 83: 1024-1028, 2007.

6. Yorimitsu T and Klionsky DJ: Autophagy: Molecular machinery for self-eating. Cell Death Differ 12 (Suppl 2): S1542-S1552, 2005.

7. Mandelbaum J, Rollins N, Shah P, Bowman D, Lee JY, Tayber O, Bernard H, LeRoy P, Li P, Koenig E, et al: Identification of a lung cancer cell line deficient in atg7-dependent autophagy. Autophagy: 0, 2015.

8. Taherbhoy AM, Tait SW, Kaiser SE, Williams AH, Deng A, Nourse A, Hammel M, Kurinov I, Rock CO, Green DR and Schulman BA: Atg8 transfer from Atg7 to Atg3: A distinctive E1-E2 architecture and mechanism in the autophagy pathway. Mol Cell 44: 451-461, 2011

9. Klionsky DJ, Abdalla FC, Abeliovich H, Abraham RT, Acevedo-Arozena A, Adeli K, Agholme L, Agnello M, Agostinis P, Aguirre-Ghiso JA, et al: Guidelines for the use and interpretation of assays for monitoring autophagy. Autophagy 8: 445-454, 2012

10. Tanida I, Ueno T and Kominami E: LC3 and autophagy. Methods Mol Biol 445: 77-88, 2008.

11. Brech A, Ahlquist T, Lothe RA and Stenmark H: Autophagy in tumour suppression and promotion. Mol Oncol 3: 366-375, 2009.

12. White E and DiPaola RS: The double-edged sword of autophagy modulation in cancer. Clin Cancer Res 15: 5308-5316, 2009.

13. Tsukada M and Ohsumi Y: Isolation and characterization of autophagy-defective mutants of Saccharomyces cerevisiae. FEBS Lett 333: 169-174, 1993.

14. Mizushima N, Yoshimori T and Ohsumi Y: The role of Atg proteins in autophagosome formation. Annu Rev Cell Dev Biol 27: 107-132, 2011.

15. Fimia GM, Stoykova A, Romagnoli A, Giunta L, Di Bartolomeo S, Nardacci R, Corazzari M, Fuoco C, Ucar A, Schwartz P, et al: Ambra1 regulates autophagy and development of the nervous system. Nature 447: 1121-1125, 2007.
16. Itakura $\mathrm{E}$, Kishi $\mathrm{C}$, Inoue $\mathrm{K}$ and Mizushima $\mathrm{N}$ : Beclin 1 forms two distinct phosphatidylinositol 3-kinase complexes with mammalian Atg14 and UVRAG. Mol Biol Cell 19: 5360-5372, 2008.

17. Zhong Y,Wang QJ,LiX, Yan Y, Backer JM, Chait BT, Heintz N and Yue Z: Distinct regulation of autophagic activity by Atg14L and Rubicon associated with Beclin 1-phosphatidylinositol-3-kinase complex. Nat Cell Biol 11: 468-476, 2009.

18. Hara T, Nakamura K, Matsui M, Yamamoto A, Nakahara Y, Suzuki-Migishima R, Yokoyama M, Mishima K, Saito I, Okano H and Mizushima N: Suppression of basal autophagy in neural cells causes neurodegenerative disease in mice. Nature 441: 885-889, 2006.

19. Klionsky DJ and Emr SD: Autophagy as a regulated pathway of cellular degradation. Science 290: 1717-1721, 2000.

20. Kroemer G, Mariño G and Levine B: Autophagy and the integrated stress response. Mol Cell 40: 280-293, 2010.

21. Xie Z and Klionsky DJ: Autophagosome formation: Core machinery and adaptations. Nat Cell Biol 9: 1102-1109, 2007.

22. Mizushima N, Noda T, Yoshimori T, Tanaka Y, Ishii T, George MD, Klionsky DJ, Ohsumi M and Ohsumi Y: A protein conjugation system essential for autophagy. Nature 395: 395-398, 1998.

23. Ichimura Y, Kirisako T, Takao T, Satomi Y, Shimonishi Y, Ishihara N, Mizushima N, Tanida I, Kominami E, Ohsumi M, et al: A ubiquitin-like system mediates protein lipidation. Nature 408: 488-492, 2000.

24. Ohsumi Y: Molecular dissection of autophagy: Two ubiquitin-like systems. Nat Rev Mol Cell Biol 2: 211-216, 2001.

25. Tanida I, Tanida-Miyake E, Ueno T and Kominami E: The human homolog of Saccharomyces cerevisiae Apg7p is a Protein-activating enzyme for multiple substrates including human Apg12p, GATE-16, GABARAP, and MAP-LC3. J Biol Chem 276: 1701-1706, 2001.

26. Komatsu M, Waguri S, Ueno T, Iwata J, Murata S, Tanida I, Ezaki J, Mizushima N, Ohsumi Y, Uchiyama Y, et al: Impairment of starvation-induced and constitutive autophagy in Atg7-deficient mice. J Cell Biol 169: 425-434, 2005.

27. Kessel D and Arroyo AS: Apoptotic and autophagic responses to Bcl-2 inhibition and photodamage. Photochem Photobiol Sci 6: 1290-1295, 2007

28. Xue LY, Chiu SM and Oleinick NL: Photochemical destruction of the Bcl-2 oncoprotein during photodynamic therapy with the phthalocyanine photosensitizer Pc 4. Oncogene 20: 3420-3427, 2001.

29. Kang R, Zeh HJ, Lotze MT and Tang D: The Beclin 1 network regulates autophagy and apoptosis. Cell Death Differ 18: 571-580, 2011.

30. Glick D, Barth S and Macleod KF: Autophagy: Cellular and molecular mechanisms. J Pathol 221: 3-12, 2010.

31. Kessel D, Vicente MG and Reiners JJ Jr: Initiation of apoptosis and autophagy by photodynamic therapy. Autophagy 2: 289-290, 2006.

32. Dewaele M, Martinet W, Rubio N, Verfaillie T, de Witte PA, Piette $\mathrm{J}$ and Agostinis P: Autophagy pathways activated in response to PDT contribute to cell resistance against ROS damage. J Cell Mol Med 15: 1402-1414, 2011.

33. Scherz-Shouval R, Shvets E, Fass E, Shorer H, Gil L and Elazar Z: Reactive oxygen species are essential for autophagy and specifically regulate the activity of Atg4. EMBO J 26: 1749-1760, 2007.

34. Azad MB, Chen Y and Gibson SB: Regulation of autophagy by reactive oxygen species (ROS): Implications for cancer progression and treatment. Antioxid Redox Signal 11: 777-790, 2009.

35. Chen Y, Azad MB and Gibson SB: Superoxide is the major reactive oxygen species regulating autophagy. Cell Death Differ 16: 1040-1052, 2009.

36. Song J, Oh Y and Lee JE: miR-Let7A modulates autophagy induction in LPS-activated microglia. Exp Neurobiol 24: 117-125, 2015. 